\title{
ON THE PARTIAL COMPACTIFICATION OF THE ARITHMETIC QUOTIENT OF A PERIOD MATRIX DOMAIN ${ }^{1}$
}

\author{
BY EDUARDO H. CATTANI
}

Communicated by S. S. Chern, August 30, 1973

In his survey paper [4], P. A. Griffiths conjectured (\$9.2) the existence of a partial compactification for the arithmetic quotients of period matrix domains. In this paper we want to announce some results concerning the topological aspects of this conjecture for the case of the periods of 2-forms on a polarized Hodge manifold $V$.

The period matrix domain $D$ of all possible period matrices for the primitive harmonic 2-forms on $V$ can be described as follows (cf. [3], [6]). Let $h=h^{2,0}, k=h^{1,1}$, where $h^{p, q}$ denotes the dimension of the space of primitive harmonic forms of bidegree $(p, q)$. Let $H$ be a complex vector space of dimension $m=2 h+k, H_{R} \subset H$ a real form, and $H_{Z} \subset H_{R}$ a lattice. We also fix a rationally defined, nondegenerate, bilinear symmetric form $Q$ on $H$. We denote by $X$ the set of all $W \in \operatorname{Gr}(h, H)$ satisfying

$$
Q(W, W)=0 .
$$

The algebraic group $G_{C}=$ orthogonal group of $Q$, acts transitively on $X$, and therefore $X$ is a projective manifold (in fact $X$ is a Kähler $C$-space, cf. [5]). The period matrix domain $D$ is the open subset of $X$ consisting of all those points which satisfy

$$
Q(W, \bar{W})>0 .
$$

(i) and (ii) are called the Riemann bilinear relations.

The noncompact real form of $G_{C}$ given by: $G=\left\{T \in G \mid T H_{R} \subset H_{R}\right\}$ acts transitively on $D$ (cf. [3]). Thus $D$ is an open, homogeneous, complex manifold of the form

$$
D=G / H=S O(2 h, k) / U(h) \times S O(k) .
$$

AMS (MOS) subject classifications (1970). Primary 32G20, 32M10; Secondary 53C30, 53C35.

Key words and phrases. Partial compactification, period matrix domain, boundary components, arithmetic subgroup, fundamental domains, Satake topology.

${ }^{1}$ This work was supported in part by the National Science Foundation, Grant No. NSF-GP-37069X. 
Let $K \subset G$ be the unique (cf. [5]) maximal compact subgroup of $G$ containing $H$. Then

$$
R=G / K=S O(2 h, k) / S O(h) \times S O(k)
$$

is a Riemannian symmetric space of the noncompact type, dual to the real Grassmannian manifold $S O(m) / S O(2 h) \times S O(k)$ (cf. [7]). Moreover $R$ can be given explicitly as

$$
R=\left\{U \in \operatorname{Gr}\left(2 h, H_{R}\right) \mid Q(U, U)>0\right\} .
$$

The natural projection $\pi: D \rightarrow R$ given by the inclusion $H \subset K$ can be described as: $\pi(W)=U$ if and only if $W \oplus \bar{W}=U+i U ; W \in D, U \in R$.

Let $\bar{D}$ (resp. $\bar{R}$ ) denote the topological closure of $D$ (resp. $R$ ) in $X$ (resp. $\left.\operatorname{Gr}\left(2 h, H_{R}\right)\right)$. Let $\partial D=\bar{D}-D, \partial R=\bar{R}-R$.

If $W_{0} \subset H$ is a totally isotropic subspace (i.e. $Q\left(W_{0}, W_{0}\right)=0$; $\left.Q\left(W_{0}, \vec{W}_{0}\right)=0\right)$, then we denote $F\left(W_{0}\right)$ by

$$
F\left(W_{0}\right)=\left\{W \in \partial D \mid W=W_{0} \oplus W_{1} ; Q\left(W_{1}, \bar{W}_{1}\right)>0\right\} .
$$

If $U_{0} \subset H_{R}$ is an even dimensional, totally isotropic subspace, then we denote $F_{D}\left(U_{0}\right)$ by $\bigcup F\left(W_{0}\right)$, where the union runs through all totally isotropic subspaces $W_{0} \subset H$ such that $W_{0} \oplus \vec{W}_{0}=U_{0}+i U_{0}$. We call $F_{D}\left(U_{0}\right)$ the preboundary component of $D$ associated to $U_{0}$. We will also denote $\mathscr{F}_{R}\left(U_{0}\right)$ by

$$
\mathscr{F}_{R}\left(U_{0}\right)=\left\{U \in \bar{R} \mid U=U_{0} \oplus U_{1} ; Q\left(U_{1}, U_{1}\right)>0\right\} .
$$

$\mathscr{F}_{R}\left(U_{0}\right)$ is called the boundary component of $R$ associated to $U_{0}$. We define an equivalence relation in $F_{D}\left(U_{0}\right)$ by: $W \sim W^{\prime}$ if and only if

$$
W+U_{0}+i U_{0}=W^{\prime}+U_{0}+i U_{0} ; W, W^{\prime} \in F_{D}\left(U_{0}\right) .
$$

$\mathscr{F}_{D}\left(U_{0}\right)=F_{D}\left(U_{0}\right) / \sim$ is called the boundary component of $D$ associated to $U_{0}$.

Finally we will denote

$$
\begin{aligned}
& N\left(\mathscr{F}_{D}\left(U_{0}\right)\right)=\left\{T \in G \mid T\left(\mathscr{F}_{D}\left(U_{0}\right)\right)=\mathscr{F}_{D}\left(U_{0}\right)\right\}, \\
& Z\left(\mathscr{F}_{D}\left(U_{0}\right)\right)=\left\{T \in G \mid T[W]=[W], \forall[W] \in \mathscr{F}_{D}\left(U_{0}\right)\right\}, \\
& G\left(\mathscr{F}_{D}\left(U_{0}\right)\right)=N\left(\mathscr{F}_{D}\left(U_{0}\right)\right) / Z\left(\mathscr{F}_{D}\left(U_{0}\right)\right) .
\end{aligned}
$$

THEOREM 1. Let $U_{0}$ be a $2 v$-dimensional totally isotropic subspace of $H_{R}$. Let $\mathscr{F}=\mathscr{F}_{D}\left(U_{0}\right)$, then:

(i) $N(\mathscr{F})$ is a parabolic subgroup of $G$ acting transitively on $\mathscr{F}$.

(ii) $G(\mathscr{F}) \cong S O(2(h-v), k-2 v)$ and therefore is a real semisimple Lie group.

(iii) $\mathscr{F}=G(\mathscr{F}) / H(\mathscr{F})$ where $H(\mathscr{F})$ is a compact subgroup of $G(\mathscr{F})$ isomorphic to $U(h-v) \times S O(k-2 v)$. 
Definition. Let $\Gamma \subset G$ be the arithmetic subgroup of $G$ defined by $\Gamma=\left\{T \in G \mid T H_{Z} \subset H_{Z}\right\}$. We say that a boundary component $\mathscr{F}_{D}\left(U_{0}\right)$ or $\mathscr{F}_{R}\left(U_{0}\right)$ is $\Gamma$-rational if the subspace $U_{0} \subset H_{R}$ is rational. We will denote by $D^{*}$ (resp. $R^{*}$ ) the union of $D$ (resp. $R$ ) and all its $\Gamma$-rational boundary components. One can prove (cf. [1], [2]) that if $\mathscr{F}_{D}\left(U_{0}\right)$ is a $\Gamma$-rational boundary component then

$$
\Gamma\left(\mathscr{F}_{D}\left(U_{0}\right)\right)=\Gamma \cap N\left(\mathscr{F}_{D}\left(U_{0}\right)\right) / \Gamma \cap Z\left(\mathscr{F}_{D}\left(U_{0}\right)\right)
$$

is an arithmetic subgroup of $G\left(\mathscr{F}_{D}\left(U_{0}\right)\right)$.

Generalizing similar results known for symmetric spaces (cf. [1], [8]) we can prove the following:

THEOREM 2. There exists an open $\Gamma$-fundamental domain $\Omega \subset D$ such that:

(i) $\bar{\Omega}$ intersects only rational preboundary components of $D$ and just a finite number of them.

(ii) If $F_{D}\left(U_{0}\right)$ is a $\Gamma$-rational preboundary component of $D$ such that $\bar{\Omega} \cap F_{D}\left(U_{0}\right) \neq \varnothing$, then $\Omega\left(\mathscr{F}_{D}\left(U_{0}\right)\right)=\bar{\Omega} \cap F_{D}\left(U_{0}\right) / \sim$ is a $\Gamma\left(\mathscr{F}_{D}\left(U_{-}\right)\right)_{0}$ fundamental domain in $\mathscr{F}_{D}\left(U_{0}\right)$.

Using Theorem 2 we can then define a Satake topology on $\Gamma \backslash D^{*}$ as in [1] and [8], and we can prove the following:

THEOREM 3. The quotient $\Gamma \backslash D^{*}$ endowed with the Satake topology has the following properties:

(i) $\Gamma \backslash D^{*}$ is locally compact and Hausdorff.

(ii) $\Gamma \backslash D \subset \Gamma \backslash D^{*}$ is an open, everywhere dense subset.

(iii) $\Gamma \backslash D^{*}$ is the finite union of subspaces of the form $\Gamma\left(\mathscr{F}_{i}\right) \backslash \mathscr{F}_{i}$ where the $\mathscr{F}_{i}$ 's run through a set of representatives of $\Gamma$-equivalence classes of $\Gamma$-rational boundary components of $D$.

(iv) The map $\pi: D \rightarrow R$ extends to a continuous map $\pi^{*}: \Gamma / D^{*} \rightarrow \Gamma \backslash R^{*}$ where $\Gamma \backslash R^{*}$ is endowed with the Satake topology.

Details and proofs will appear in a later publication.

ACKnowledgement. Most of the results announced here are contained in the author's $\mathrm{Ph}$.D. thesis, written under the direction of Professor W. M. Boothby at Washington University. The author also wishes to thank Professor S. Kobayashi for many valuable suggestions.

\section{REFERENCES}

1. W. L. Baily, Jr. and A. Borel, Compactification of arithmetic quotients of bounded symmetric domains, Ann. of Math. (2) 84 (1966), 442-528. MR 35 \#6870.

2. E. Cattani, On the partial compactification of the "modular variety" associated to a Kähler surface, Thesis, Washington University, 1972. 
3. P. A. Griffiths, Periods of integrals on algebraic manifolds. I, II, Amer. J. Math. 90 (1968), 586-626; 805-865. MR 37 \#5215; 38 \#2146.

4. - Periods of integrals on algebraic manifolds: Summary of main results and discussion of open problems, Bull. Amer. Math. Soc. 76 (1970), 228-296. MR 41 \#3470.

5. P. A. Griffiths and W. Schmid, Locally homogeneous complex manifolds, Acta Math. 123 (1969), 253-302. MR 41 \#4587.

6. - Recent developments in Hodge theory: a discussion of techniques and results (to appear).

7. S. Helgason, Differential geometry and symmetric spaces, Pure and Appl. Math., vol. 12, Academic Press, New York, 1962. MR 26 \#2986.

8. I. Satake, On compactification of the quotient spaces for arithmetically defined discontinuous groups, Ann. of Math. (2) 72 (1960), 555-580. MR 30 \#594.

Courant Institute of Mathematical Sciences, New York University, New YORK, NeW YORK 10012

Current address: Department of Mathematics and Statistics, University of Massachusetts, Amherst, Massachusetts 01002 